\title{
Vitamin B12 and Vitamin D Levels in Patients with Autoimmune Hypothyroidism and Their Correlation with Anti-Thyroid Peroxidase Antibodies
}

\author{
Hanife Şerife Aktaş \\ Health Sciences University, Umraniye Training and Research Hospital, Internal Medicine, Istanbul, Turkey
}

\section{Significance of the Study}

- There was a negative correlation between vitamin B12 and vitamin D levels and anti-thyroid peroxidase antibodies in patients with autoimmune hypothyroidism.

\section{Keywords}

Vitamin B12 - Vitamin D . Autoimmune hypothyroidism .

Anti-thyroid peroxidase antibodies

\section{Abstract}

Background: This study was designed to investigate the vitamin D (vit-D) and vitamin B12 (vit-B12) levels and their correlation with anti-thyroid peroxidase (anti-TPO) antibodies in patients with autoimmune hypothyroidism. Methods: A total of 130 patients diagnosed with autoimmune hypothyroidism were included in the study retrospectively. The patients were divided into two groups as those having vit-B12 levels below $200 \mathrm{pg} / \mathrm{mL}(n=60)$ and vit-B12 levels equal to or above $200 \mathrm{pg} / \mathrm{mL}(n=70)$. These two groups were compared in terms of age, sex, thyroid-stimulating hormone (TSH), free-T4 (FT4), vit-D, and anti-TPO. The correlation between vit-B12 and anti-TPO levels was also investigated in these groups. Patients were then divided into four groups according to their vit-D levels. Patients with normal vit-D levels $(25[\mathrm{OH}] \mathrm{D}>30 \mathrm{ng} / \mathrm{mL} ; n=5)$, those with vit-D insufficiency $(20-30 \mathrm{mg} / \mathrm{mL} ; n=9)$, those with vit-D deficiency (10-20 ng/
$\mathrm{mL} ; n=43)$, and those with severe vit-D deficiency ( $<10 \mathrm{ng} /$ $\mathrm{mL} ; n=73$ ). These four groups were compared in terms of age, gender, TSH, FT4, vit-B12, and anti-TPO levels. In addition, the correlation between levels of vit-D and anti-TPO was also investigated. Results: We found that vit-B12 deficiency and vit-D deficiency were associated with autoimmune hypothyroidism, and that there was a negative correlation between vit-B12 and vit-D levels and anti-TPO antibodies in these patients. Conclusion: In patients with autoimmune hypothyroidism, vit-D and vit-B12 deficiency should be investigated at the time of diagnosis and periodically on follow-ups.

(c) 2019 The Author(s) Published by S. Karger AG, Basel

\section{Introduction}

Hashimoto thyroiditis (HT) is the most common cause of hypothyroidism, a disease caused by inadequate synthesis and/or release of thyroid hormones [1]. In these patients, hypothyroidism at various levels is accompanied by autoantibodies (antithyroid peroxidase antibody 
[anti-TPO], antithyroglobulin antibody) and lymphocytic infiltration in thyroid tissue. It is more common in the 30 - to 50 -year-old age group and is $4-10$ times more common in women than in men [2].

Environmental (30\%) and genetic (70\%) factors influence the etiopathogenesis of HT [3]. Genetic factors include the major histocompatibility gene (HLA), immune regulator genes (CTLA4, PTPN22, FOXP3, CD25, CD40, and FRCL3) and thyroid-specific genes (TSHR, Tg) [3]. The possible environmental factors in etiology are smoking, alcohol, dietary iodine, stress, selenium, vitamin D (vit-D) deficiency, bacterial and viral infections, pregnancy, and drugs [4].

The main function of vit-D is to achieve calcium and phosphorus balance with bone metabolism. In recent years, however, vit-D has been reported to have a role in the formation of autoimmune diseases, heart diseases, cancer, inflammatory bowel diseases, diabetes, and rheumatologic diseases, as well as its effects on bone metabolism [5]. In addition, it is thought that vit-D may play a role in the regulation of the immune system because vit- $\mathrm{D}$ receptors are detected on cells of the immune system [6]. In some studies, a relationship between vit-D deficiency and autoimmune thyroid diseases such as HT and Grave's disease has been reported, and impaired vit-D signaling has been reported in thyroid cancers [7]. Bozkurt et al. [8] reported that vit-D deficiency had a role in the development of HT.

Many autoimmune diseases, such as pernicious anemia, myasthenia gravis, idiopathic hypoparathyroidism, vitiligo, celiac disease, type 1 diabetes mellitus, autoimmune liver diseases, primary biliary cirrhosis, multiple sclerosis, Addison's disease, rheumatoid arthritis, and systemic lupus erythematosus, may accompany HT [9]. Vitamin B12 (vit-B12) deficiency has been reported frequently in autoimmune thyroid patients [10]. This association is probably due to impaired absorption of vit-B12 by atrophic gastritis and/or pernicious anemia associated with autoimmune thyroid disease [11]. Atrophic gastritis is seen in $35-40 \%$ of autoimmune thyroid diseases [12]. It was reported that $1 / 3$ of the patients with primary hypothyroidism had antibodies against gastric parietal cells and $12 \%$ had pernicious anemia. Intrinsic factor antibodies have also been shown in these patients [13].

$\mathrm{HT}$ is an autoimmune disease and studies suggest that vit-D deficiency has an effect on the etiopathogenesis of the disease. In addition, vit-B12 deficiency is frequently seen in this disease $[7,10]$. This study was designed to investigate levels of vit-D and vit-B12 in patients with autoimmune hypothyroidism and to evaluate their correlation with anti-TPO antibodies.

Vit-B12 and Vit-D, Hypothyroidism, and Anti-TPO Antibodies
Table 1. Demographic data and distribution of parameters

\begin{tabular}{lc}
\hline Male & $15(11.5)$ \\
Female & $115(88.5)$ \\
Vit-B12 $<200 \mathrm{pg} / \mathrm{mL}$ & $60(46)$ \\
Vit-D $<30 \mathrm{ng} / \mathrm{mL}$ & $125(96)$ \\
Anti-TPO $(+)$ & $130(100)$ \\
Age, years & \\
$\quad$ Mean & $41.4 \pm 1.9$ \\
$\quad$ Median & $41(18-64)$ \\
\hline
\end{tabular}

Values are presented as $n(\%)$, the mean $\pm \mathrm{SD}$, or median (min.$\max$.).

\section{Materials and Methods}

A total of 130 patients aged between 18 and 65 years who were diagnosed with autoimmune hypothyroidism in the Internal Medicine Outpatient Clinic of Umraniye Education and Research Hospital were included in the study retrospectively. Approval was obtained from the Ethics Committee of our hospital. Exclusion criteria were thyroidectomy, receiving RAI treatment, malabsorption syndromes, gastrectomy, ileal resection, a vegetarian diet, pregnancy, and use of drugs to treat vit-B12 deficiency (phenytoin, metformin, and DHF reductase inhibition).

The patients were divided into two groups as those having vit-B12 levels below $200 \mathrm{pg} / \mathrm{mL}(n=60)$ and vit-B12 levels equal to or above $200 \mathrm{pg} / \mathrm{mL}(n=70)$ [14]. These two groups were compared in terms of age, sex, thyroid-stimulating hormone (TSH), free-T4 (FT4), vit-D, and anti-TPO. In addition, the correlation between levels of vit-B12 and anti-TPO was also examined in these two groups. Patients were then divided into four groups according to their vit-D levels [5]: patients with normal vit-D levels $(25[\mathrm{OH}] \mathrm{D}>30 \mathrm{ng} / \mathrm{mL} ; n=5)$, those with vit-D insufficiency $(20-30 \mathrm{ng} / \mathrm{mL} ; n=9)$, those with vit-D deficiency $(10-20 \mathrm{ng} / \mathrm{mL} ; n=43)$, and those with severe vit-D deficiency $(<10 \mathrm{ng} / \mathrm{mL} ; n=73)$. Then the age, gender, TSH, FT4, vit-B12, and anti-TPO levels of these four groups were compared. In addition, the correlation between vit-D and anti-TPO levels was also investigated in these four groups.

Serum vit-B12 (187-883 pg/mL), vit-D (>30 ng/mL), TSH (0.35-4.94 mU/mL), FT4 $(0.4-1.48 \mathrm{ng} / \mathrm{mL})$, and anti-TPO $(<5.6$ $\mathrm{IU} / \mathrm{mL}$ ) measurements were performed in the fasting blood taken from the patients on the same day. These tests were performed by ABBOTT Architect i2000 with the chemiluminescence method. The anti-TPO antibody cut off level was accepted as $5.61 \mathrm{IU} / \mathrm{mL}$.

\section{Statistical Analysis}

Statistical analyses were performed using the MedCalc Statistical Software version 12.7.7 (MedCalc Software bvba, Ostend, Belgium; http://www.medcalc.org; 2013). Statistical significance was set at $p<0.05$. Descriptive statistics were used to define continuous variables (mean \pm standard deviation, and median, minimum and maximum). The comparison of two independent groups with a normal distribution was performed using the Student $t$ test. The comparison of two independent groups without a normal distribution was performed using the Mann-Whitney U test. The correla- 
Table 2. Relationship between vit-B12 levels and TSH, FT4, anti-TPO, and vit-D

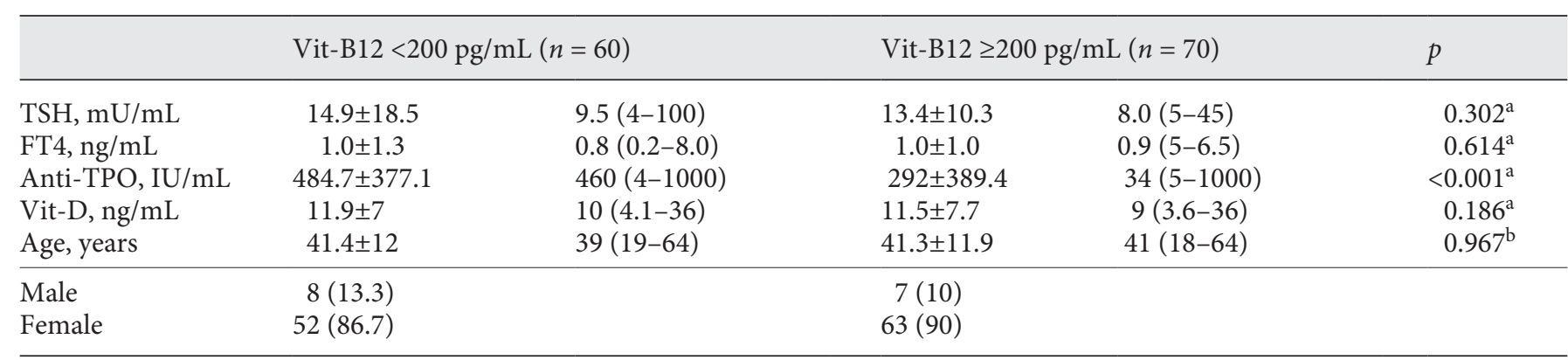

Data are presented as the mean $\pm \mathrm{SD}$, median (min.- max.), and $n(\%)$.

a Mann-Whitney U Test. ${ }^{\mathrm{b}}$ Student $t$ test.

Fig. 1. Correlation between anti-TPO and vit-B12. There was a weak, negative, statistically significant correlation between antiTPO and vit-B12 levels in both groups with and without vit-B12 deficiency $(r=-0.394$, $p<0.001)$

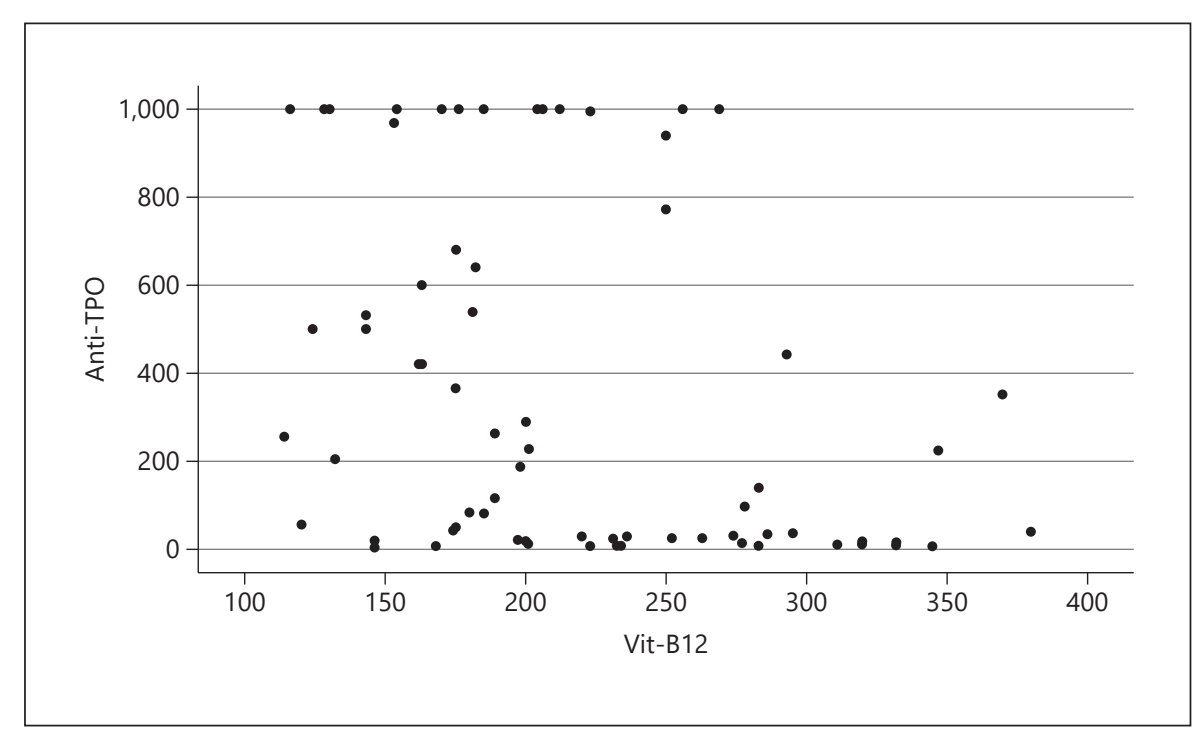

tion between two continuous variables not compatible with a normal distribution was examined with the Spearman rho correlation coefficient. Statistical evaluation of categorical variables was performed using the $\chi^{2}$ test.

\section{Results}

The mean age of all patients was $41.4+11.9$ years, and 115 patients $(88.5 \%)$ were female and $15(11.5 \%)$ were male. Vit-B12 deficiency was found in 60 (46\%) and vit-D deficiency was found in 125 (96.1\%) of all patients. Seventy-three patients (56.1\%) with hypothyroidism had severe vit-D deficiency. Anti-TPO antibody was positive in all patients (Table 1 ).
There was no significant difference between the two vit-B12 groups in terms of age, gender, TSH, FT4, and vit$\mathrm{D}$ levels. However, the anti-TPO levels were significantly higher in patients with low vit-B12 levels $(p<0.001$; Table $2)$. There was no significant difference between the four vit-D groups in terms of age, gender TSH, FT4, vit-B12, and anti-TPO levels (Table 3).

There was a weak, negative correlation between TSH and vit-D levels in the $25(\mathrm{OH}) \mathrm{D}<10 \mathrm{ng} / \mathrm{mL}$ group. Moreover, there was also a weak, negative correlation between anti-TPO and vit-D levels in the $25(\mathrm{OH}) \mathrm{D}<10 \mathrm{ng} / \mathrm{mL}$ group. There was a weak, negative correlation between anti-TPO and vit-B12 levels in all patient groups $(p<$ 0.001; Table 4; Fig. 1). There was also a weak, negative correlation between anti-TPO and vit-D levels in all of the groups (Table 5; Fig. 2). 


\section{Discussion}

HT is the most common cause of primary hypothyroidism. The disease is more common in the 30- to 50year age group, and $95 \%$ of patients are women. The female to male ratio is about 7.2 [15]. In our study, the male to female ratio was 7.7 , and the mean age of the patients was $41.4 \pm 11.9$ years, similar to the literature.

There are several studies suggesting that the prevalence of vit-D deficiency is high in patients with $\mathrm{HT}$ and that there is a relationship between hypothyroidism and vit-D in these patients [16]. In our study, we found that $96 \%$ of patients with HT had vit-D deficiency, and $56.1 \%$ of patients had severe vit-D deficiency $(<10 \mathrm{ng} / \mathrm{mL})$. We also found a negative correlation between vit-D levels and anti-TPO levels in these patients. We think that vit-D deficiency may cause autoimmune hypothyroidism. Bozkurt et al. [8] reported that vit-D deficiency had a role in the development of HT.

Some studies have reported a relationship between vit$\mathrm{D}$ deficiency and thyroid autoimmunity in all age groups, and decreased anti-TPO levels when cholecalciferol supplementation is performed in HT patients with vit-D deficiency $[17,18]$. Kivity et al. [19] reported that the prevalence of vit-D deficiency $(25[\mathrm{OH}] \mathrm{D}$ level $<25 \mathrm{nmol} / \mathrm{L})$ was higher in people with autoimmune thyroid disease. They also reported a correlation between vit-D deficiency and anti-TPO levels and that this had an effect on the pathogenesis of autoimmune thyroid diseases. Mansournia et al. [20] reported a significant negative correlation between HT disease and serum levels of 25(OH)D. Shin et al. [21] reported that people with high anti-TPO levels had lower vit-D levels.

Mazokopakis et al. [17] showed that serum levels of $25(\mathrm{OH}) \mathrm{D}$ were inversely related to anti-TPO levels in patients with HT. Anti-TPO levels were also significantly higher in HT patients with vit-D deficiency $(<75 \mathrm{nmol} / \mathrm{L})$ than those HT patients without vit-D deficiency. In another study, a significant decrease in serum anti-TPO levels was reported after 4 months of oral vit-D3 supplementation (1,200-4,000 IU/day) in patients with vit-D deficiency [17].Screening for vit-Ddeficiency and replacement therapy if necessary has been recommended [22]. Levels of vit-D were observed to be significantly lower in children with HT [23]. A negative correlation between vit-D and TPO antibodies was found in our study. HT disease activity has been reported to decrease with vit-D treatment [24].

The relationship between vit-D deficiency and thyroid autoimmunity remains unclear. However, it is suggested

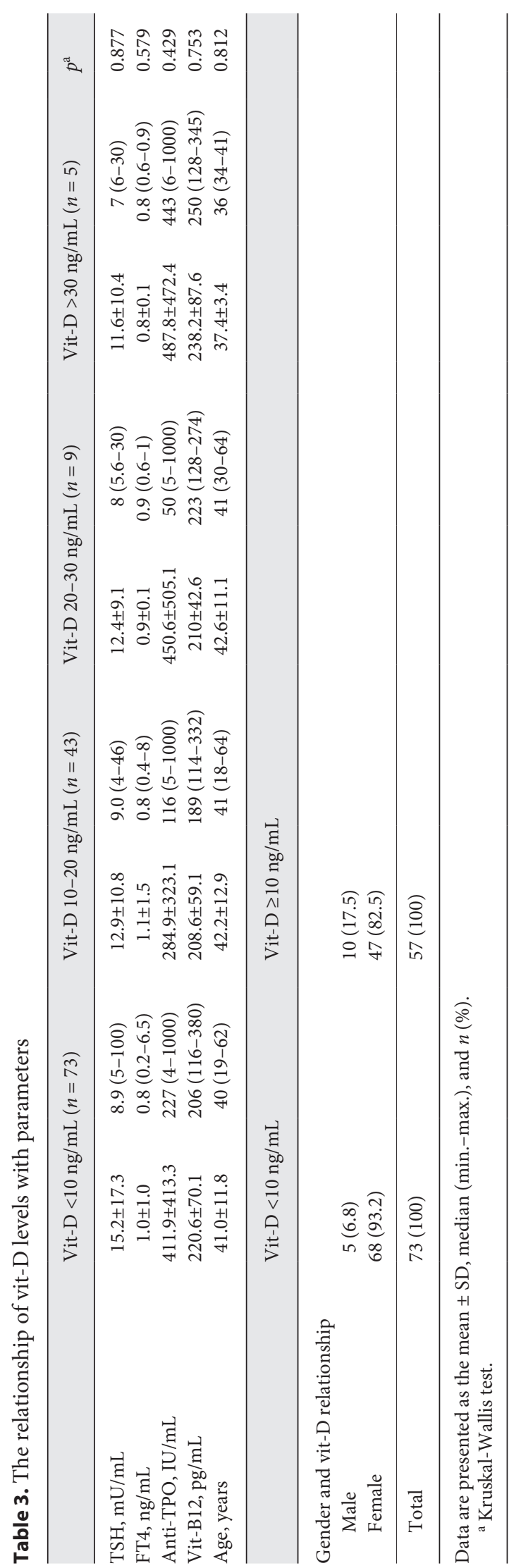


Table 4. Correlation between levels of vit-B12 and other parameters

\begin{tabular}{lrr}
\hline & $r^{\mathrm{a}}$ & $p$ \\
\hline Vit-B12 $<200 \mathrm{pg} / \mathrm{mL}(n=60)$ & & \\
TSH and B12 & -0.182 & 0.164 \\
Anti-TPO and B12 & -0.170 & 0.194 \\
FT4 and B12 & -0.055 & 0.674 \\
Vit-B12 $\geq 200 \mathrm{pg} / \mathrm{mL}(n=70)$ & & \\
TSH and B12 & -0.102 & 0.401 \\
Anti-TPO and B12 & -0.170 & 0.159 \\
FT4 and B12 & 0.062 & 0.609 \\
All groups & & \\
TSH and B12 & 0.018 & 0.835 \\
Anti-TPO and B12 & -0.394 & $<0.001$ \\
FT4 and B12 & 0.039 & 0.658 \\
\hline
\end{tabular}

${ }^{a}$ Spearman rho correlation coefficient.
Table 5. Correlation between vit-D and other parameters

\begin{tabular}{lrl}
\hline & $r^{\mathrm{a}}$ & $p$ \\
\hline Vit-D $<10$ ng/mL $(n=73)$ & & \\
TSH and vit-D & -0.304 & 0.009 \\
Anti-TPO and vit-D & -0.257 & 0.028 \\
FT4 and vit-D & 0.177 & 0.134 \\
Vit-D $\geq 10$ ng/mL $(n=57)$ & & \\
TSH and vit-D & -0.021 & 0.876 \\
Anti-TPO and vit-D & 0.067 & 0.619 \\
FT4 and vit-D & 0.005 & 0.969 \\
All groups & & \\
TSH and vit-D & -0.149 & 0.091 \\
Anti-TPO and vit-D & -0.185 & 0.035 \\
FT4 and vit-D & 0.068 & 0.441 \\
\hline
\end{tabular}

a Spearman rho correlation coefficient.
Fig. 2. Correlation between anti-TPO and vit-D. There was also a weak, negative, and statistically significant correlation between anti-TPO and vit-D levels in all these groups $(r=-0.185, p=0.035)$.

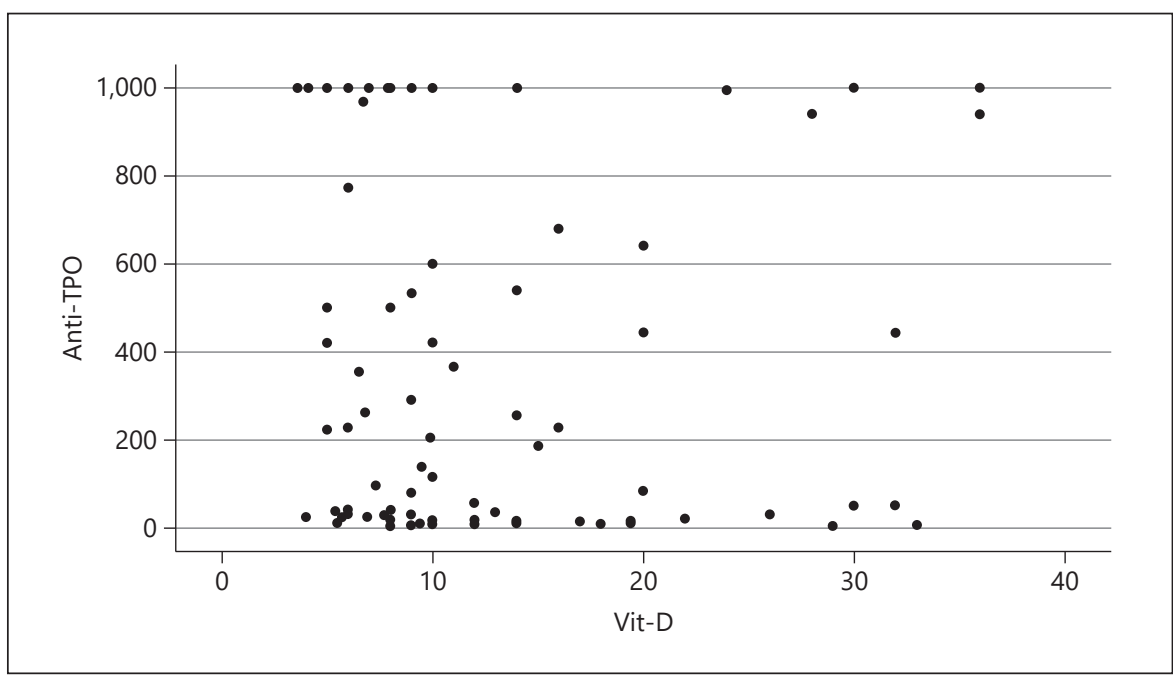

that vit-D may be associated with anti-inflammatory and immunomodulatory effects. Vit-D plays a significant role in modulation of the immune system, enhancing the innate immune response while exerting an inhibitory action on the adaptive immune system. Most immune cells, including $\mathrm{T}$ cells, B cells, dendritic cells, and macrophages, express the vit-D receptor and 1a-hydroxylase [25].

In our study, we found that the incidence of vit-B12 deficiency in autoimmune hypothyroid patients was $46 \%$. We also found that there was a negative correlation between anti-TPO and vit-B12 levels. It is possible that this may be due to pernicious anemia or atrophic gastritis accompanying autoimmune hypothyroidism, which is often accompanied by other autoimmune diseases. Perni- cious anemia may be part of a polyglandular endocrinopathyassociated with hypothyroidism [26]. This association is caused by atrophic gastritis and/or pernicious anemia associated with autoimmune thyroid diseases disrupting vit-B12 absorption. Overall, $35-40 \%$ of patients with autoimmune thyroid diseases have atrophic gastritis [26], while $33 \%$ of patients with primary hypothyroidism have been shown to have antibodies to gastric parietal cells, and $12 \%$ have pernicious anemia. Intrinsic factor antibodies have also been observed in patients with autoimmune hypothyroidism [9].

The prevalence of vit-B12 deficiency in patients with hypothyroidism in Pakistan, Turkey, and India is reported to be $40.5,18.6$, and $10 \%$, respectively [27]. The differences in prevalence between these ratios could be caused 
by differences in diet and nutrition status. Clinical manifestations of vit-B12 deficiency are very variable. Hematological and neurological abnormalities may coexist, be independently found, or be very uncertain. Even in asymptomatic patients, the first sign may be the detection of vit-B12 deficiency [28]. Patients with both hypothyroidism and vit-B12 deficiency also have similar symptoms. Symptoms such as fatigue, weakness, dementia, depression, memory impairment, lethargy, and tingling are frequently seen in patients with hypothyroidism and vitB12 deficiency. Thus, vit-B12 deficiency may be ignored in hypothyroidism.

Vit-B12 deficiency increases homocysteine levels [13] and a relationship is known to exist between hyperhomocysteinemia and the development of atherosclerosis. Several studies have shown a relationship between hypothyroidism and homocysteine levels. Thus, if vit-B12 deficiency is overlooked in hypothyroid patients it may increase cardiovascular risk through hyperhomocysteinemia [29].

Ness-Abramof et al. [30] recommend screening vitB12 levels at the time of diagnosis of autoimmune thyroid disease, with 3- to 5-year intervals. Collins and Pawlak [28] also recommend screening for vit-B12 deficiency in hypothyroid patients at the time of diagnosis and at certain intervals periodically. Our study supports these recommendations.

One of the limitations of this study is its retrospective design. Another is the small number of patients included. Studies investigating the relationship between future hypothyroidism and vit-D and vit-B12 should be prospectively designed in larger sample sizes.

\section{Conclusion}

In this study, we observed vit-B12 and vit-D deficiency in patients with HT. We also found a negative correlation between vit-D deficiency, vit-B12 deficiency, and levels of anti-TPO. We suggest that vit-D deficiency contributes to the etiopathogenesis of HT, and that vit-B deficiency and HT are part of a polyglandular endocrine pathology. Vit$\mathrm{D}$ and vit-B12 deficiency should be investigated in patients with autoimmune hypothyroidism at the time of diagnosis and during periodic follow-ups, and supplementation should be performed in those who need it. More randomized controlled trials are needed to investigate the effect of vit-D and vit-B12 deficiency in the etiopathogenesis of HT, and to determine the effect of replacement therapy on the clinical course of HT.

\section{Statement of Ethics}

All procedures performed in this study involving human participants were in accordance with the ethical standards of the institutional and/or national research committee and with the 1964 Helsinki declaration and its later amendments or comparable ethical standards.

\section{Disclosure Statement}

All authors declare that they have no conflicts of interest. No financial support was obtained.

\section{References}

1 Caturegli P, De Remigis A, Rose NR. Hashimoto thyroiditis: clinical and diagnostic criteria. Autoimmun Rev. 2014 Apr-May;13(4-5): 391-7.

2 McLeod DS, Caturegli P, Cooper DS, Matos PG, Hutfless S. Variation in rates of autoimmune thyroid disease by race/ethnicity in US military personnel. JAMA. 2014 Apr;311(15): $1563-5$.

3 Wiersinga WM. Clinical relevance of environmental factors in the pathogenesis of autoimmune thyroid disease. Endocrinol Metab. 2016;31(2):213.

4 Liontiris MI, Mazokopakis EE. A concise review of Hashimoto thyroiditis (HT) and the importance of iodine, selenium, vitamin D and gluten on the autoimmunity and dietary management of HT patients.Points that need more investigation. Hell J Nucl Med. 2017 Jan-Apr;20(1):51-6.
5 Holick MF. Vitamin D deficiency. N Engl J Med. 2007;357:266-81.

6 Bikle D. Nonclassic actions of vitamin D. J Clin Endocrinol Metab. 2009 Jan;94(1):2634.

7 Muscogiuri G, Tirabassi G, Bizzaro G, Orio F, Paschou SA, Vryonidou A, et al. Vitamin D and thyroid disease: to D or not to D? Eur J Clin Nutr. 2015 Mar;69(3):291-6.

8 Bozkurt NC, Karbek B, Ucan B, Sahin M, Cakal E, Ozbek M, et al. The association between severity of vitamin D deficiency and Hashimoto's thyroiditis. Endocr Pract. 2013 May-Jun;19(3):479-84.

9 Dilas LT, Icin T, Paro JN, Bajkin I. Autoimmune thyroid disease and other non-endocrine autoimmune diseases. Med Pregl. 2011 Mar-Apr;64(3-4):183-7. Serbian.
10 Lippi G, Montagnana M, Targher G, Salvagno GL, Guidi GC. Prevalence of folic Acid and vitamin $\mathrm{B} 12$ deficiencies in patients with thyroid disorders. Am J Med Sci. 2008 Jul;336(1): $50-2$.

11 Iddah MA, Macharia BN. Autoimmune thyroid disorders. ISRN Endocrinol. 2013 Jun; 2013:509764.

12 Lahner E, Centanni M, Agnello G, Gargano L, Vannella L, Iannoni C, et al. Occurrence and risk factors for autoimmune thyroid disease in patients with atrophic body gastritis. Am J Med. 2008 Feb;121(2):136-41.

13 Jaya Kumari S, Bantwal G, Devanath A, Aiyyar V, Patil M. Evaluation of serum vitamin B12 levels and its correlation with anti-thyroperoxidase antibody in patients with autoimmune thyroid disorders. Indian J Clin Biochem. 2015 Apr;30(2):217-20.
Vit-B12 and Vit-D, Hypothyroidism, and Anti-TPO Antibodies
Med Princ Pract 2020;29:364-370

DOI: $10.1159 / 000505094$ 
14 Allen LH. Vitamin B-12. Adv Nutr. 2012 Jan; 3(1):54-5.

15 Nalbant A, Aydın A, Tilla H, Cinemre H. Hashimoto tiroiditli olguların klinik ve laboratuvar bulgularınındeğerlendirilmesi. Online Türk Sağlık Bilimleri Dergisi. 2016;1(3): 8-20.

16 Arslan MS, Topaloglu O, Ucan B, Karakose M, Karbek B, Tutal E, et al. Isolated vitamin D deficiency is not associated with nonthyroidal illness syndrome, but with thyroid autoimmunity. ScientificWorldJournal. 2015;2015: 239815.

17 Mazokopakis EE, Papadomanolaki MG Tsekouras KC, Evangelopoulos AD, Kotsiris $\mathrm{DA}$, Tzortzinis AA. Is vitamin $\mathrm{D}$ related to pathogenesis and treatment of Hashimoto's thyroiditis? Hell J Nucl Med. 2015 Sep-Dec; 18(3):222-7.

18 Chaudhary S, Dutta D, Kumar M, Saha S, Mondal SA, Kumar A, et al. Vitamin D supplementation reduces thyroid peroxidase antibody levels in patients with autoimmune thyroid disease: an open-labeled randomized controlled trial. Indian J Endocrinol Metab. 2016 May-Jun;20(3):391-8.
19 Kivity S, Agmon-Levin N, Zisappl M, Shapira Y, Nagy EV, Dankó K, et al. Vitamin D and autoimmune thyroid diseases. Cell Mol Immunol. 2011 May;8(3):243-7.

20 Mansournia N, Mansournia MA, Saeedi S, Dehghan J. The association between serum 25OHD levels and hypothyroid Hashimoto's thyroiditis. J Endocrinol Invest. 2014 May; 37(5):473-6.

21 Shin DY, Kim KJ, Kim D, Hwang S, Lee EJ. Low serum vitamin $\mathrm{D}$ is associated with antithyroid peroxidase antibody in autoimmune thyroiditis. Yonsei Med J. 2014 Mar;55(2): 476-81.

22 Caprio $\mathrm{M}$, Infante $\mathrm{M}$, Calanchini $\mathrm{M}$ et al. Vitamin D: not just the bone. Evidence for benecial pleiotropic extraskeletal effects. Eat Weight Disord. 2017;22(1):27-41.

23 Sönmezgöz E, Ozer S, Yilmaz R, Önder Y, Bütün I, Bilge S. Hypovitaminosis D in children with Hashimoto"s thyroiditis. Rev Med Chil. 2016 May;144(5):611-6. Spanish.

24 Chahardoli R, Saboor-Yaraghi AA, Amouzegar A, Khalili D, Vakili AZ, Azizi F. Can Supplementation with Vitamin D Modify Thyroid Autoantibodies (Anti-TPO Ab, Anti-Tg $\mathrm{Ab}$ ) and Thyroid Profile (T3, T4, TSH) in Hashimoto's Thyroiditis? A Double Blind, Randomized Clinical Trial. Horm Metab Res. 2019 May;51(5):296-301.
25 Aranow C. Vitamin D and the immune system. J Investig Med. 2011 Aug;59(6):881-6.

26 Jabbar A, Yawar A, Waseem S, Islam N, Ul Haque N, Zuberi L, et al. Vitamin B12 deficiency common in primary hypothyroidism. J Pak Med Assoc. 2008 May;58(5):258-61.

27 Das C, Sahana PK, Sengupta N, Giri D, Roy M, Mukhopadhyay P. Etiology of anemia in primary hypothyroid subjects in a tertiary care center in eastern india. Indian J Encorinol Metab. 2012;16:S361-3.

28 Collins AB, Pawlak R. Prevalence of vitamin B-12 deficiency among patients with thyroid dysfunction. Asia Pac J Clin Nutr. 2016;25: 221-226.

29 Lien EA, Nedrebø BG, Varhaug JE, Nygård O, Aakvaag A, Ueland PM. Plasma total homocysteine levels during short-term iatrogenic hypothyroidism. J Clin Endocrinol Metab. 2000 Mar;85(3):1049-53.

30 Ness-Abramof R, Nabriski DA, Braverman LE, Shilo L, Weiss E, Reshef T, et al. Prevalence and evaluation of B12 deficiency in patients with autoimmune thyroid disease. Am J Med Sci. 2006 Sep;332(3):119-22. 